\title{
An observational revisit of band-split solar type-II radio bursts
}

\author{
Guohui $\mathrm{Du}^{1}$,Xiangliang $\mathrm{Kong}^{1}$,Yao Chen ${ }^{1}$, Shiwei Feng ${ }^{1}$, Bing Wang ${ }^{1}$, and Gang $\mathrm{Li}^{2}$
}

\begin{abstract}
Band split of solar type II radio bursts, discovered several decades ago, is a fascinating phenomenon with the type-II lanes exhibiting two almost-parallel sub-bands with similar morphology. The underlying split mechanism remains elusive. One popular interpretation is that the splitting bands are emitted from the shock upstream and downstream, respectively, with their frequency ratio $(\gamma)$ determined by the shock compression ratio. This interpretation has been taken as the physical basis for many published references. Here we report an observational analysis of type II events with nice split selected from the ground-based RSTN data from 2001 to 2014, in the metric-decametric wavelength. We investigate the temporal variation and distribution of $\gamma$, and conduct correlation analyses on the deduced spectral values. It is found that $\gamma$ varies in a very narrow range with $>80 \%$ of $\gamma$ (one-minute averaged data) being between 1.15 to 1.25. For some well-observed and long-lasting events, $\gamma$ does not show a systematic variation trend within observational uncertainties, from the onset to the termination of the splits. In addition, the parameters representing the propagation speed of the radio source (presumably the coronal shock) show a very weak or basically no correlation with $\gamma$. We suggest that these results do not favor the upstreamdownstream scenario of band splits.
\end{abstract}

Subject headings: Sun: coronal mass ejections (CMEs) — Sun: corona — shock waves - Sun: radio radiation

\section{Introduction}

Solar type II radio bursts are excited by energetic electrons accelerated during coronal eruptions (Payne-Scott et al. 1947; Wild et al. 1963; Nelson \& Melrose 1985). It is generally

\footnotetext{
${ }^{1}$ Shandong Provincial Key Laboratory of Optical Astronomy and Solar-Terrestrial Environment, and Institute of Space Sciences, Shandong University, Weihai 264209, China; yaochen@sdu.edu.cn

${ }^{2}$ Department of Space Science and CSPAR, University of Alabama in Huntsville, Huntsville, AL 35899, USA
} 
believed that coronal shocks are accelerators of these electrons (see, Chen et al. 2014; Feng et al. 2015; Kong et al. 2015, for latest studies). Despite intensive studies over half a century, it has not been completely understood how these electrons are accelerated at shocks, and how the radio emissions are excited.

One fascinating feature of solar type II radio bursts is the band split (McLean 1967; Wild \& Smerd 1972; Smerd et al. 1974). This feature can appear on both the fundamental and harmonic branches. The physical mechanism underlying this phenomenon remains unresolved during the past decades. Proposed scenarios have been briefly reviewed in Du et al. (2014), and can be divided into two classes. In the first class it is assumed that the split is caused by the shock structure, such as the upstream, downstream, as well as the transition region across the shock (Smerd et al. 1974, 1975), or the shock interaction with coronal structures at different locations characterized by different emission frequencies (McLean 1967). In the other class of scenarios it is assumed that the split is associated with some intrinsic emission mechanism (e.g., Treumann \& Labelle 1992). It should be pointed out that none of the available scenarios receives solid observational or theoretical supports, so the exact mechanism of band splits remains unknown. Nevertheless, in many published references, the assumption that the split bands are respectively emitted from downstream (the upper band with higher frequency) and the upstream (the lower band with lower frequency) has been used for shock diagnostics, for example, to infer the compression ratio and the shock Mach number, etc. (e.g., Smerd et al. 1974; Vršnak et al. 2002, 2004; Cho et al. 2007; Liu et al. 2009; Ma et al. 2011; Vasanth et al. 2014; Zucca et al. 2014; Long et al. 2015). However, the upstream-downstream (UD) scenario suffers from the absence of energetic electrons and/or enhanced Langmuir oscillations in the shock downstream, either theoretically or observationally, (see Cairns 2011 for a recent review).

Thus, it is important to search for observational clues and physical mechanisms accounting for the band-split phenomenon. In a series of studies, Vršnak et al. $(2002,2004)$ investigated statistically the corona and interplanetary type-II bursts with band split. Assuming the UD scenario, they further deduced the magnitude and radial profile of the Alfvén speed and magnetic field strength. Differences of their studies and ours will be discussed later in this paper. In this study we present an observational revisit of band-split type II events by examining the radio spectral data from the ground-based RSTN (Radio Solar Telescope Network, operated by the US Air Force) in the metric to decametric wavelength. 


\section{Events selection and their general properties}

The type-II band-split (BS) events are selected according to their spectral characteristics, and we focus on the ground-based observational data that are in the metric to decametric wavelength. Following Du et al. (2014), we require that the two bands should be narrow and clear enough with similar intensity and morphology variations, and without strong interfer-

ence from other type of solar bursts or artificial signals. In addition, the frequency ratio of the upper and lower bands should be less than 2 to avoid confusion with the fundamental and harmonic branches (see also, Vršnak et al. 2001). We also require that the events should last for more than one minute. We examine the RSTN data from 2000 to 2014, and select 18 candidate events.

Mostly according to the lifetime and clearness of the band splits, we further separate the events into two groups. Group A events have a lifetime longer than 10 minutes with generally nice BS features. This allows us to examine the temporal evolution of the split parameters during an individual event. Events in group B either have a shorter lifetime or their BS features are not as clear as those of group A events.

The spectra of group A events are given in Figure 1, and some basic parameters of all the events (referred to as Group A+B) have been listed in Table 1 including the accompanying CME and flare properties. For further quantitative analysis, we have over-plotted the backbones of the splitting bands with dashed lines. These backbones are given by the intensity maximum. Only the better-observed branches are superposed with their backbones. In further studies, we will measure the exact values of these frequencies and calculate the average frequency drift and the split ratio. The frequencies will be divided by 2 if they are taken from the harmonic branch.

In Table 1, the first three columns show the event dates, the start and end times of the type II emission, and the interval used in this study which is selected to avoid data with unclear split feature or strong interference. Further spectral parameters given in the following columns are deduced within this selected interval. The start and end frequencies of the lower band $\left(f_{\text {start }}\right.$ and $\left.f_{\text {end }}\right)$, the average drift (Df/Dt), and the average band-split frequency ratio $(\gamma)$ are given in the fourth to sixth columns. The first appearance time of the coronal mass ejection (CME) in the LASCO-C2/SOHO (Domingo et al. 1995) field of view, and the flare data are given in the left columns. The CME and flare data with superscripts are obtained by the authors while others are taken from the CDAW-CME catalogue (Yashiro et al. 2004) and the SolarSoft Latest Events Archive (http : //www.lmsal.com/solarsoft/latest_events_archive.html). Note that in Events 20140220 and 20140907, two episodes of split bands can be identified, and we list them separately in Table 1. 
The most striking feature of Figure 1 is the similarity of the frequency ratio of the two splitting bands for different events. Note that these events are observed during different phase of the solar cycle, with significant difference in the spectral drift and CME-flare parameters. This can also be seen from Table 1 that the average frequency split ratio varies from 1.14 to 1.29 while the average drift varies in a large range from -0.03 to $-0.23 \mathrm{MHz} \mathrm{s}^{-1}$. In addition, the CME linear speeds also vary significantly from $\sim 200 \mathrm{~km} \mathrm{~s}^{-1}$ to $\sim 1300 \mathrm{~km} \mathrm{~s}^{-1}$, and the flare levels vary from C1.1 to X1.0.

Comparing the timing data given in Table 1, we find that for all events, the type II starting time is earlier than the CME first appearance time in LASCO C2, and later than the flare start times. In addition, the type II bursts start within 5 minutes of the flare peaking time for 12 events. This is consistent with previous statistical correlation studies of the type II bursts and solar eruptions (e.g., Mancuso et al. 2004). It is not possible to infer the exact cause (i.e., CME or flare) of the radio bursts only with these timing data.

To further illustrate the similarity of different BS events, in Figure 2(a) we superpose the backbones of the eight events in Group A. In doing so, we take the two-fold (2X) Saito density model (Saito 1970) as the reference model. Data on each backbone are moved in time as a whole until a minimum deviation from the backbone to the 2X Saito model is achieved. We see that all these temporally-shifted backbones, being put together, still exhibit a recognizable BS morphology. This highlights the obvious spectral similarity among different data sets.

In Figure 2(b), we plot the frequency ratios $(\gamma)$ of the splitting bands together with the same starting time. Two features should be emphasized. Firstly, a majority of $\gamma$ lies in a narrow range of 1.15 to 1.25 with only a few outliers. Secondly, we find that within observational uncertainties $\gamma$ does not vary much in time for individual events. To show the uncertainty of the data, we determine the frequency profiles given by $90 \%$ of the intensity maximum for one specific event (20020125) which is long enough to cover most part of the frequency range. This produces four spectral lines. We then take the ratios of the outer two lines and the inner two lines to be the maximum and the minimum of the $\gamma$ uncertainty. Other events have similar uncertainty range, and will not be shown here for clearness. These two features, i.e., the values of $\gamma$ do not change significantly from event to event and during the evolution of a specific event, are consistent with previous studies on fewer and earlier events (see, e.g., Vršnak et al. 2001). We confirm this finding with more events in this study.

To take into account the large spectral changes during an event, we calculate the oneminute averaged values of $\gamma$. To do this, the data are separated into one-minute long episodes with adjacent ones having an overlap of 30 seconds. For an event lasting for 5 minutes, this produces 9 data points of the averaged $\gamma$. Histograms of the number of data points with the 
one-minute averaged values of $\gamma$ for Group A and Group A+B are given in Figure 2(c) and Figure 2(d), respectively. We see that for both data sets, the mean and median values of $\gamma$ are $\sim 1.2$, and the standard deviation $(\mathrm{SD})$ is 0.03 for Group A and 0.04 for Group A+B. For Group A $(\mathrm{A}+\mathrm{B}), \sim 95(80) \%$ of the data points lie in a narrow range of $[1.15,1.25]$. This result is consistent with the conclusion obtained above.

\section{Correlation analysis of the splitting frequency ratio $(\gamma)$ and other spectral parameters}

It is generally believed that the type II radio bursts are given by plasma emission with the emission frequency mainly determined by the electron density at the radio source (Ginzberg \& Zhelezniakov 1958). So the spectra data of type II bursts can be used to deduce the source velocity using a density model of the corona. Among various density models, the Saito (Saito 1970) and Newkirk (Newkirk 1961) models have been widely used in relevant studies. The $2 \mathrm{X}$ Saito model is very close to the Newkirk model in the inner corona, and both models are represented with multinomial function of the radial distance.

Similarly, we make a simplified assumption that the corona density and the type-II frequency can be approximated by the following power laws of the radial distance (see also Gopalswamy et al. 2009),

$$
n=n_{0} r^{-\alpha}, f=f_{0} r^{-\frac{\alpha}{2}}
$$

where $n_{0}$ and $f_{0}$ are the number density and plasma frequency at the coronal base. Assuming the radio source (presumably the coronal shock) velocity is $v_{s}$, we have

$$
\begin{aligned}
& \frac{D f}{D t}=-\frac{v_{s}}{2} \alpha f_{0}^{-\frac{2}{\alpha}} \cdot f^{\frac{\alpha+2}{\alpha}} \\
& v_{s} \propto-\frac{2}{\alpha} \frac{D f}{D t} f^{-\frac{\alpha+2}{\alpha}}=v_{s}^{p}
\end{aligned}
$$

From previous studies, it is well known that the type-II spectral drift is strongly correlated with the emission frequency. This indicates that lower in the corona the density gradient is larger and thus the spectral drift is higher, in general (e.g., Vršnak et al. 2001; Gopalswamy et al. 2009). This relationship has been confirmed by our data analysis shown in the upper panels of Figure 3, where we present the one-minute averaged spectral drift

$\left(\frac{D f}{D t}\right)$ versus $f^{\frac{\alpha+2}{\alpha}}$ with $\alpha$ set to $2,4,6$, and 10 in different panels for all events. We also plotted the linear fitting results with black lines. The obtained correlation coefficients $c$ for different values of $\alpha$ are around 0.67 and very close to each other, consistent with previous studies. 
From Equation (3) we can deduce a proxy of the radio source speed $\left(v_{s}^{p}\right)$ with the spectral data if given the power law index $\alpha$. We then check the correlation of this source speed proxy with the band split frequency ratio $\gamma$. The results are given in the lower panels of Figure 3 for different assumptions of $\alpha$. In each panel we also present the linear fitting line and the corresponding correlation coefficient $(c)$. It is found that in panel $(\mathrm{e}) c=0.2$ for $\alpha=2$, while for other values of $\alpha c$ is less than 0.1 . This indicates a general weak or even no correlation between the shock speed proxy and the type-II split ratio.

In Figure 4, we plot the deduced radio-source speed with the 2X Saito density model by fitting the radio spectra to the lower band of the type II splits. Again, we separate the data into episodes of one minute with adjacent episodes sharing 30 seconds of data. The correlations of the obtained source speeds and the frequency split ratios of the events in Group A and Group A+B are given in panels (a) and (b). The correlation coefficients are only marginally larger than 0.1 indicating very weak correlations. The weak or even no correlation described above is another major result of this study.

\section{Conclusions and discussion}

With the RSTN radio spectral data, we collected 18 type II events with nice band-split features. The striking similarities of the splitting bands and the relative constant frequency ratio of the two splitted bands are highlighted. It is found that a majority $(>80 \%)$ of the split frequency ratio data lie in a very narrow range from 1.15 to 1.25 , which does not show a considerable systematic change during most individual events, within measurement uncertainty. In addition, we find that there exist very weak or basically no correlation between the prescribed proxy of the source velocity and the frequency ratio of the split bands. These results, based on the following discussion, do not favor the mostly-used UD assumption of the type-II band splits.

According to the UD scenario, the band-split ratio should strongly depend on the shock properties. These properties are determined by the coupling between the coronal eruption and the background environment. During the progression of the solar cycle, the coronal environment changes significantly, and the solar eruptions also exhibit a large variation (see our Table 1). Combining these two varying factors, it is expected that the shock properties vary significantly from event to event. Furthermore, when the UD scenario was first proposed by Smerd et al. (1974), the shock was considered to be an ideal discontinuity. In reality, in the shock upstream and downstream, strong turbulence as well as the shock foot and overshoot structures may develop (Li et al. 2013; Schwartz et al. 2011; Scudder et al. 1986). This certainly adds more variation effect to the upstream-downstream density ratio. Therefore, 
if the UD scenario is correct, at least the following three outcomes shall be expected.

Firstly, the density ratio shall present a large variation from event to event. In addition, from the onset to the termination of a specific type-II event, the shock may evolve significantly and so likely does the density compression. These expectations are not observed in our data analysis. Secondly, the split frequency (presumably the density compression ratio according to the UD scenario) should strongly depend on the shock properties. The shock itself is considered to be related to a weak diffusive structure in the coronagraph and possibly associated with the EUV wave front in the EUV imaging data (see, e.g., Chen et al. 2014; Feng et al. 2015), whose properties are still very difficult to measure. So a direct examination of the shock compression measurement and the band-split ratio is very difficult, if not impossible. In this study, we presented a correlation analysis of the frequency split ratio and a velocity proxy of the radio-emitting source. The proxy is deduced using the prescribed power-law coronal density model and the frequently-used Saito density model. No correlation or only marginal correlation between the source velocity proxy and the split ratio is found. This does not lend to a strong support to the UD scenario.

Thirdly, with the UD scenario one is able to deduce the shock compression ratio with the obtained frequency split ratio. The deduced compression ratio is in general very small and lies in a narrow range of $[1.2,1.7]$. This suggests that the split is highly selective over the compression ratio, corresponding to relatively weak shocks. This result is not consistent with the intuitive expectation that energetic electrons as well as the type-II bursts are more likely associated with stronger shocks (e.g., Wu 1984).

Another evidence against the UD scenario was presented by Du et al. (2014), who found a BS event with spectral features carried by the high-frequency band appearing seconds earlier than those carried by the low-frequency band. According to Du et al., these spectral features were related to density structures which were swept by the shock during its propagation. Assuming the UD scenario, the density structures shall first appear in the shock upstream then in the downstream and relevant spectral features shall first appear on the low-frequency band then on the high-frequency band, inconsistent with the data.

As mentioned, earlier statistical studies on band splits of both the metric and interplanetary type II bursts have been presented by Vršnak et al. (2002, 2004). They also revealed the almost-constant values of the split frequency ratio. Under the UD scenario, they deduced the shock Mach number which was found not to vary much either. Their main purpose was to infer the magnitudes and radial profiles of the magnetic field strength $(\mathbf{B})$ from the corona to the interplanetary space through the following procedure.

First, the shock compression ratio is obtained with the observed split width. Then, the 
Mach number is calculated with the Rankine-Hugoniot jump conditions, assuming a specific plasma $\beta$ and a shock geometry (being parallel or perpendicular). Another step is to get the shock velocity by fitting the spectra using the prescribed density model. After this, the Alfvén speed and further the magnetic field strength can be obtained with the same density model. Through this procedure, they deduced reasonable profiles of $\mathbf{B}$, which are consistent with other diagnostic results (see Vršnak et al. 2002). It seems that this may provide a support to their working assumption that is the UD scenario. However, we point out that the above process involves many free parameters and suffers from large uncertainty. For example, the shock speed is determined by fitting the spectra with the specified density model. And it is well known that this fitting process works properly only when the radio source moves outwards along the density gradient. Also it is a fact that the corona is highly time-varying and structured and can hardly be described by a fixed density model.

In addition, since the obtained compression ratio as well as the deduced Mach number does not change much under the UD scenario, the radial profile of $\mathbf{B}$ is actually controlled by other factors. The main contribution should be the density profile, which is used to determine the shock speed by fitting the dynamic spectra and to deduce $\mathbf{B}$ from the obtained Alfvén speed. The reason that the $\mathbf{B}$ profile looks reasonable is mainly because that the adopted density model is reasonable.

Thus we suggest that the seemingly-reasonable magnitude and radial profile of B deduced with the UD scenario contains large uncertainty, the profile is mainly determined by the density dependence over distance as well as the type-II spectral drift. It should not be taken as an evidence to support the UD scenario.

In summary, the result of our study, together with that of Du et al. (2014), do not favor the UD scenario, which however has been used in many studies for coronal shock diagnostics, as mentioned. The exact physical mechanism accounting for the type II band splits remains unknown. The seemingly constancy of the frequency split ratio from event to event and during an individual event tend to suggests that the band splits are given intrinsically by the emission mechanism and not associated with outside geometrical profiles, which vary too much to account for the observation. The split mechanism, whatever it is, shall be able to confine the frequency split ratio within a relatively narrow range. Further theoretical and observational studies are demanded before a definite conclusion can be reached.

We are grateful to the RSTN, SOHO/LASCO, GOES and SolarSoft Latest Events Archive teams for making their data available to us. We thank Prof. Ching-Sheng Wu for helpful suggestion. This work was supported by grants NSBRSF 2012CB825601, NNSFC 41274175, 41331068 and the Natural Science Foundation of Shandong Province (ZR2013DQ004 
and ZR2014DQ001). Gang Li's work at UAHuntsivlle was supported by NSF grants ATM0847719 and AGS1135432.

\section{REFERENCES}

Cairns, I. H. 2011, in The Sun, the Solar Wind, and the Heliosphere, ed. M. P. Miralles \& J. S. Almeida (Berlin: Springer), 267

Chen, Y., Du, G. H., Feng, L., et al. 2014, ApJ, 787, 59

Cho, K. S., Lee, J., Moon, Y. J., et al. 2007, A\&A, 461, 1121

Domingo, V., Fleck, B. \& Poland, A. I. 1995, SoPh, 162, 1

Du, G. H., Chen, Y., Lv, M. S., et al. 2014, ApJL, 793L, 39

Feng, S. W., Du, G. H., Chen, Y., et al. 2015, Sol. Phys., 290, 1195

Gopalswamy, N., Thompson, W. T., Davila, J. M., et al. 2009, SoPh, 259, 227

Kong, X. L., Chen, Y., Guo, F., et al. 2015, ApJ., 798, 81

Li, G., Kong, X. L., Zank, G., \& Chen, Y. 2013, ApJ,769, 22

Liu, Y., Luhmann, J. G., Bale, S. D., \& Lin, R. P. 2009, ApJL, 691, L151

Long, D. M., Baker, D., Williams, D. R., et al. 2015, ApJ, 799, 224

Ma, S., Raymond, J. C., Golub, L., et al. 2011, ApJ, 738, 160

S. Mancuso., \& J. C. Raymond, 2004, A\&A, 363, 371

McLean, D. J. 1967, Proc. Astron. Soc. Australia, 1, 47

Nelson, G. S., \& Melrose, D. B. 1985, in Solar Radiophysics, ed. D. J. McLean \& N. R. Labrum (Cambridge: Cambridge Univ. Press), 333

Newkirk, G. Jr. 1961, ApJ, 133, 983

Payne-Scott, R., Yabsley, D. E., \& Bolton, J. G. 1947, Natur, 160, 256

Saito, K. 1970, Ann. Tokyo Astron. Obs., 12, 53

Schwartz, S. J., Henley, E., Mitchell, J., \& Krasnoselskikh, V. 2011, PRL, 107, 215002 
Scudder, J. D., Aggso, T. L., Mangeney, A., Lacombe, C., \& Harvey, C. C. 1986, JGR, 91, 11053

Smerd, S. F., Sheridan, K. V., \& Stewart, R. T. 1974, in IAU Symp. 57, Coronal Disturbances, ed. G. A. Newkirk (Dordrecht: Reidel), 389

Smerd, S. F., Sheridan, K. V., \& Stewart, R. T. 1975, Astrophys. Lett., 16, 23

Treumann, R. A., \& Labelle, J. 1992, ApJ, 399, L167

Vasanth, V., Umapathy, S., Vršnak. B., et al. 2014, Sol. Phys., 289, 251

Vršnak, B., Aurass, H., Magdalenić, J., \& Gopalswamy, N. 2001, A\&A, 377, 321

Vršnak, B., Aurass, H., Magdalenić, J., \& Mann, G. 2002, A\&A, 396, 673

Vršnak, B., Magdalenić, J., \& Zlobec, P. 2004, A\&A, 413, 753

Wild, J. P., Smerd, S. F., \& Weiss, A. A. 1963, ARA\&A, 1, 291

Wild, J. P., \& Smerd, S. F. 1972, ARA\&A, 10, 159

Wu, C. S., 1984, JGR, 89, 8857

Yashiro, S., N. Gopalswamy., G. Michalek., et al. 2004, JGR, 109, A07105

Zucca, P., Pick, M., Dmoulin, P., et al. 2014, ApJ., 795, 68 
Table 1: Some basic parameters of the type-II events. See text for details.

\begin{tabular}{|c|c|c|c|c|c|c|c|c|c|c|}
\hline Events & $\begin{array}{c}\text { Start-end } \\
\text { times of } \\
\text { type-IIs }\end{array}$ & $\begin{array}{c}\text { Selected } \\
\text { interval of } \\
\text { study }\end{array}$ & $\begin{array}{c}\text { Start-end } \\
\text { frequen- } \\
\text { cies } \\
\text { (lower } \\
\text { band, } \\
\mathrm{MHz} \text { ) }\end{array}$ & $\begin{array}{c}\mathrm{Df} / \mathrm{Dt} \\
(\mathrm{MHz} \\
/ \mathrm{s})\end{array}$ & $\gamma$ & $\begin{array}{c}\text { Fitting } \\
\text { source } \\
\text { speed } \\
(\mathrm{km} / \mathrm{s})\end{array}$ & $\begin{array}{c}\mathrm{CME} \\
\text { linear } \\
\text { speed } \\
(\mathrm{km} / \mathrm{s})\end{array}$ & $\begin{array}{c}\text { First C2 } \\
\text { appear- } \\
\text { ance } \\
\text { time }\end{array}$ & $\begin{array}{c}\text { Flare } \\
\text { (source) }\end{array}$ & $\begin{array}{c}\text { Flare start } \\
\& \text { peak } \\
\text { times }\end{array}$ \\
\hline \multicolumn{11}{|c|}{ Group A } \\
\hline 20010126 & $06: 05-06: 15$ & 06:09-06:14 & $64-40$ & -0.07 & 1.19 & 555 & 314 & $06: 30$ & M1(N10E63)* & $05: 50-06: 06^{*}$ \\
\hline 20020125 & $02: 26-02: 45$ & $02: 27-02: 38$ & $65-35$ & -0.05 & 1.19 & 400 & 213 & $03: 30$ & C7(N13E10)* & $02: 10-02: 26^{*}$ \\
\hline 20020511 & 11:32-11:48 & 11:36-11:41 & $48-36$ & -0.04 & 1.19 & 429 & 235 & $12: 26$ & M1.5(S06W31)* & 11:16-12:06* \\
\hline 20021004 & $22: 44-23: 00$ & $22: 47-22: 56$ & $60-34$ & -0.04 & 1.19 & 407 & 310 & $23: 30$ & M2.9(N11E43) & $22: 35-22: 43$ \\
\hline 20030709 & $16: 34-16: 53$ & $16: 42-16: 52$ & $58-39$ & -0.03 & 1.23 & 273 & $\mathrm{~N} / \mathrm{A}$ & $\mathrm{N} / \mathrm{A}$ & C5.8(N13W88) & 16:24-16:37 \\
\hline 20031118 & 07:47-08:07 & $07: 49-07: 53$ & $80-56$ & -0.10 & 1.16 & 506 & 1223 & 08:06 & M3.2(N01E19) & $07: 23-07: 52$ \\
\hline 20100613 & $05: 38-05: 58$ & $05: 40-05: 43$ & $63-44$ & -0.13 & 1.24 & 937 & 320 & $06: 06$ & M1.0(S24W82) & $05: 30-05: 39$ \\
\hline 20140108 & 03:48-03:58 & $03: 50-03: 53$ & $73-52$ & -0.14 & 1.22 & 804 & 643 & $04: 12$ & M3.6(N11W88) & 03:39-03:47 \\
\hline \multicolumn{11}{|c|}{ Group B } \\
\hline 20040604 & 07:44-08:00 & $07: 47-07: 48$ & $44-38$ & -0.14 & 1.21 & 1473 & 1306 & $07: 50$ & $\mathrm{~N} / \mathrm{A}$ & $\mathrm{N} / \mathrm{A}$ \\
\hline 20050319 & $07: 07-07: 16$ & $07: 10-07: 12$ & $52-48$ & -0.06 & 1.22 & 442 & 369 & $07: 36$ & C1.1(S19W52) & 06:24-07:06 \\
\hline 20060430 & 01:39-02:02 & $01: 40-01: 41$ & $75-56$ & -0.23 & 1.26 & 1163 & 428 & 02:06 & C5.4(N16E74) & 01:33-01:57 \\
\hline 20071231 & 00:53-01:11 & $00: 54-00: 58$ & $83-54$ & -0.11 & 1.28 & 550 & 995 & $01: 31$ & C8.8(S08E81) & 00:37-01:11 \\
\hline 20111117 & $07: 28-07: 31$ & $07: 28-07: 30$ & $74-55$ & -0.15 & 1.18 & 826 & 458 & $07: 48$ & C6.0(S19E08) & $07: 16-07: 27$ \\
\hline 20120118 & $23: 22-23: 32$ & $23: 26-23: 29$ & $58-45$ & -0.03 & 1.14 & 630 & 270 & $23: 48$ & C5.1(N25W44) & $22: 57-23: 20$ \\
\hline 20120806 & 04:44-04:48 & $04: 44-04: 46$ & $61-47$ & -0.08 & 1.15 & 590 & 198 & $05: 12$ & M1.6(S14E88) & 04:33-04:38 \\
\hline 20131119 & $10: 25-10: 34$ & $10: 27-10: 32$ & $76-48$ & -0.09 & 1.27 & 533 & 740 & $10: 36$ & X1.0(S13W69) & 10:14-10:26 \\
\hline 2014022001 & $03: 22-03: 24$ & $03: 23-03: 25$ & $52-39$ & -0.09 & 1.18 & 803 & $410^{*}$ & 04:00* & C3.3(S13E36) & 03:15-03:35 \\
\hline 2014022002 & 03:23-03:26 & 03:22-03:24 & $53-44$ & -0.08 & 1.17 & 640 & $410^{*}$ & $04: 00^{*}$ & C3.3(S13E36) & 03:15-03:35 \\
\hline 2014090701 & 02:01-02:03 & 02:01-02:02 & 95-77 & -0.23 & 1.16 & 735 & 487 & $02: 24$ & C7.5(S21E63) & 01:53-02:04 \\
\hline 2014090702 & 02:03-02:06 & $02: 03-02: 06$ & $75-53$ & -0.14 & 1.17 & 784 & 487 & $02: 24$ & C7.5(S21E63) & 01:53-02:04 \\
\hline
\end{tabular}



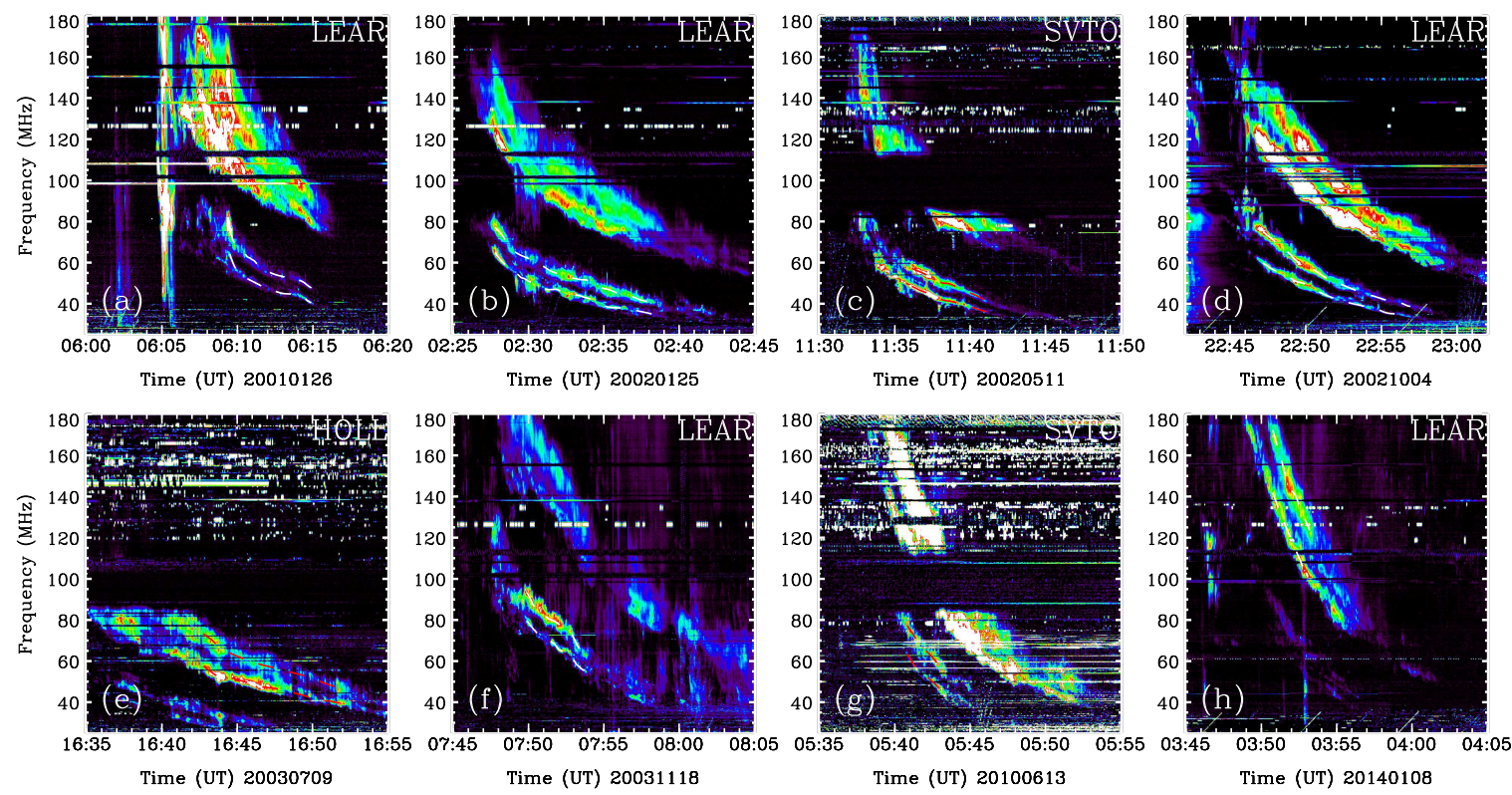

Fig. 1. - The dynamic spectra observed by the RSTN network for group A events. Dashed lines are backbones given by the intensity maximum. 

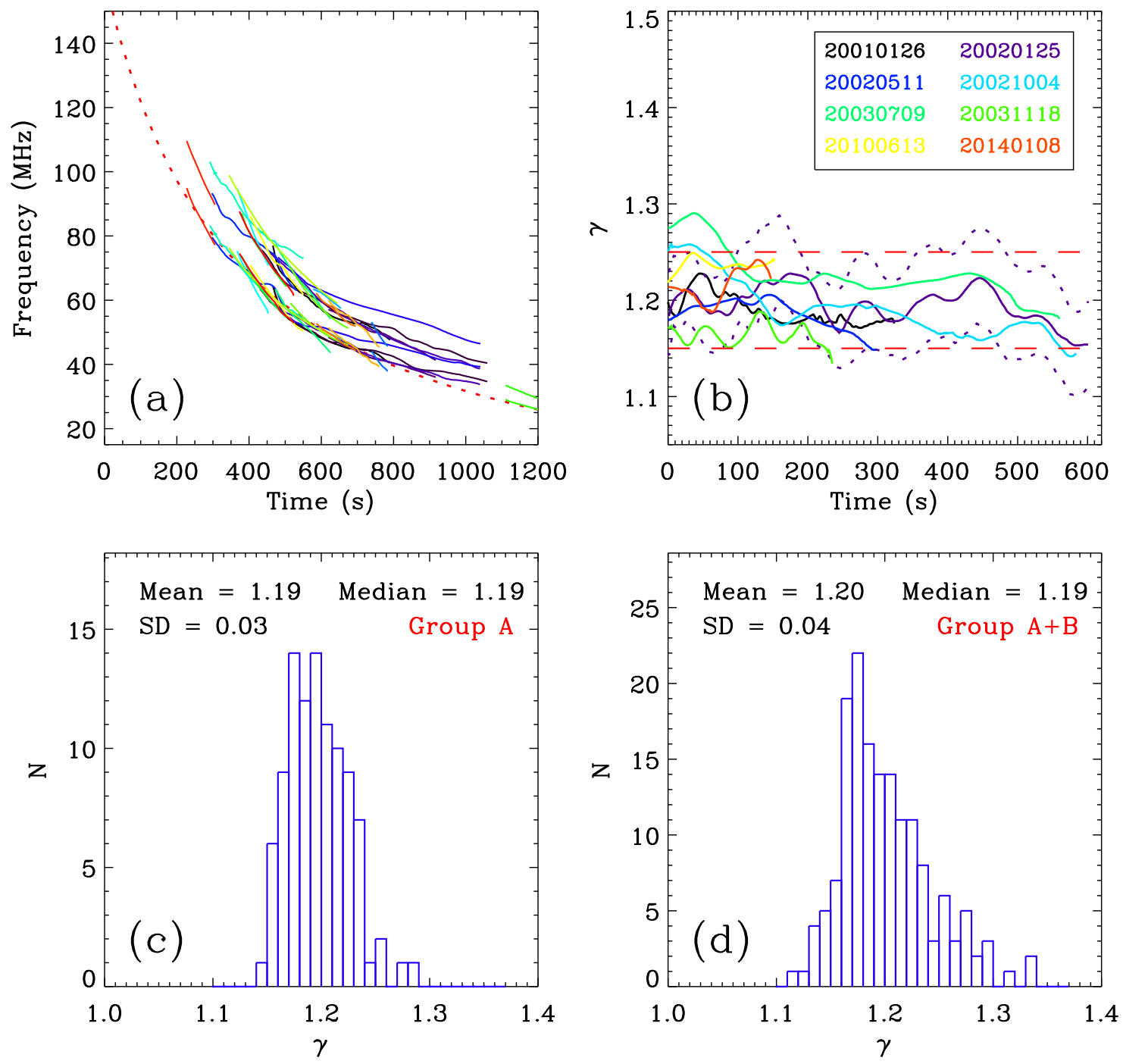

Fig. 2.- (a) Spectral backbones of Group A events plotted together with the 2X Saito density model (the dashed line). (b) Temporal evolution of band-split frequency ratios $(\gamma)$ with a fixed starting time. The two dashed lines are measurement uncertainty for Event 20020125 (see text for details). (c) and (d) are histograms of the number of data points versus the one-minute averaged values of $\gamma$ for Group A and Group A+B events. 


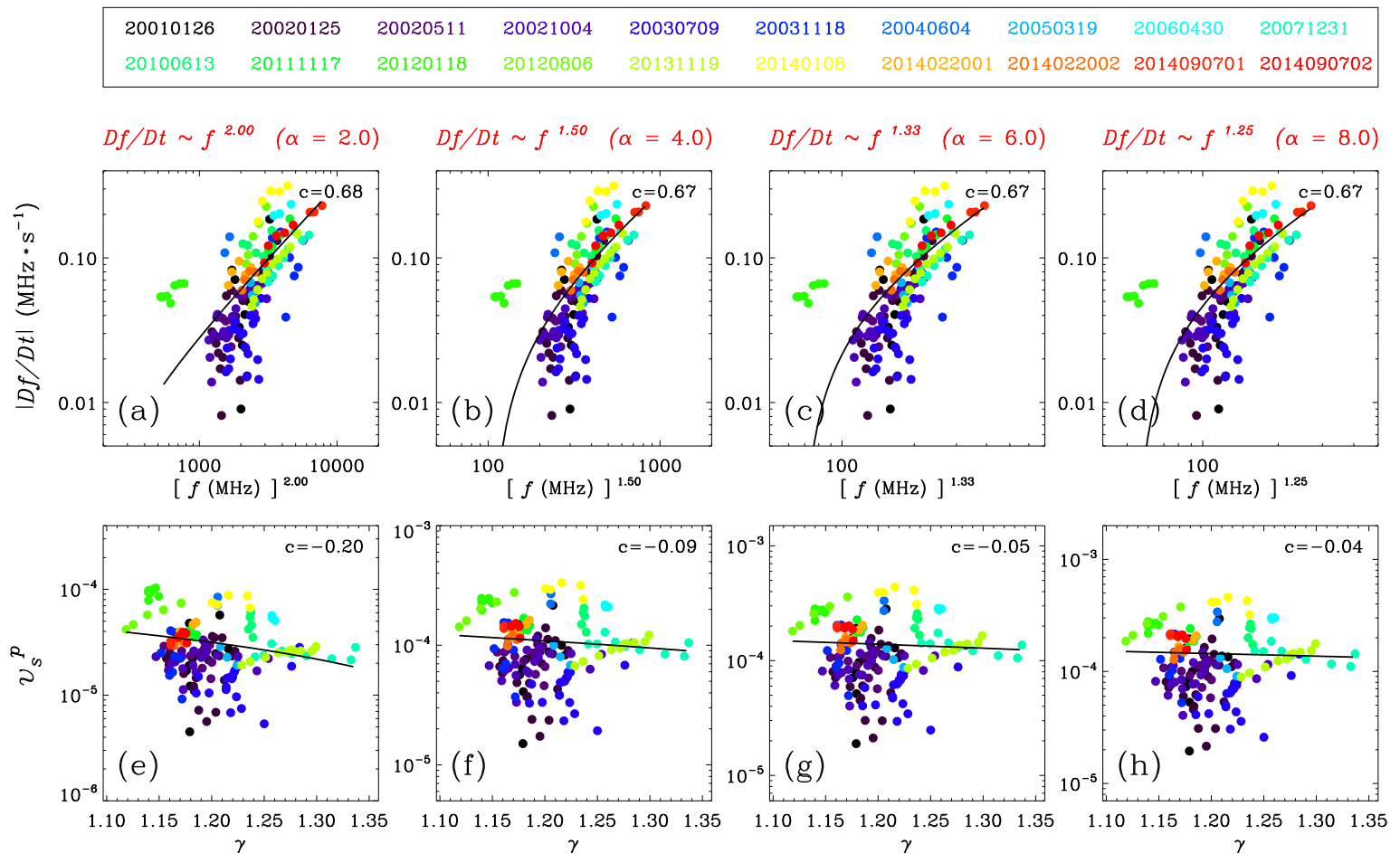

Fig. 3.- (a)-(d) One-minute averaged spectral drift $\left(\frac{D f}{D t}\right)$ versus $f^{\frac{\alpha+2}{\alpha}}$ with $\alpha$ set to $2,4,6$, and 10 for all events of our study. (e)-(h) Deduced shock velocity proxy $\left(v_{s}^{p}\right)$ versus band-split frequency ratio $\gamma$ for different values of $\alpha$ (written on top of each column). Linear fitting lines (solid black) and corresponding correlation coefficients $c$ are oresented in each panel. 

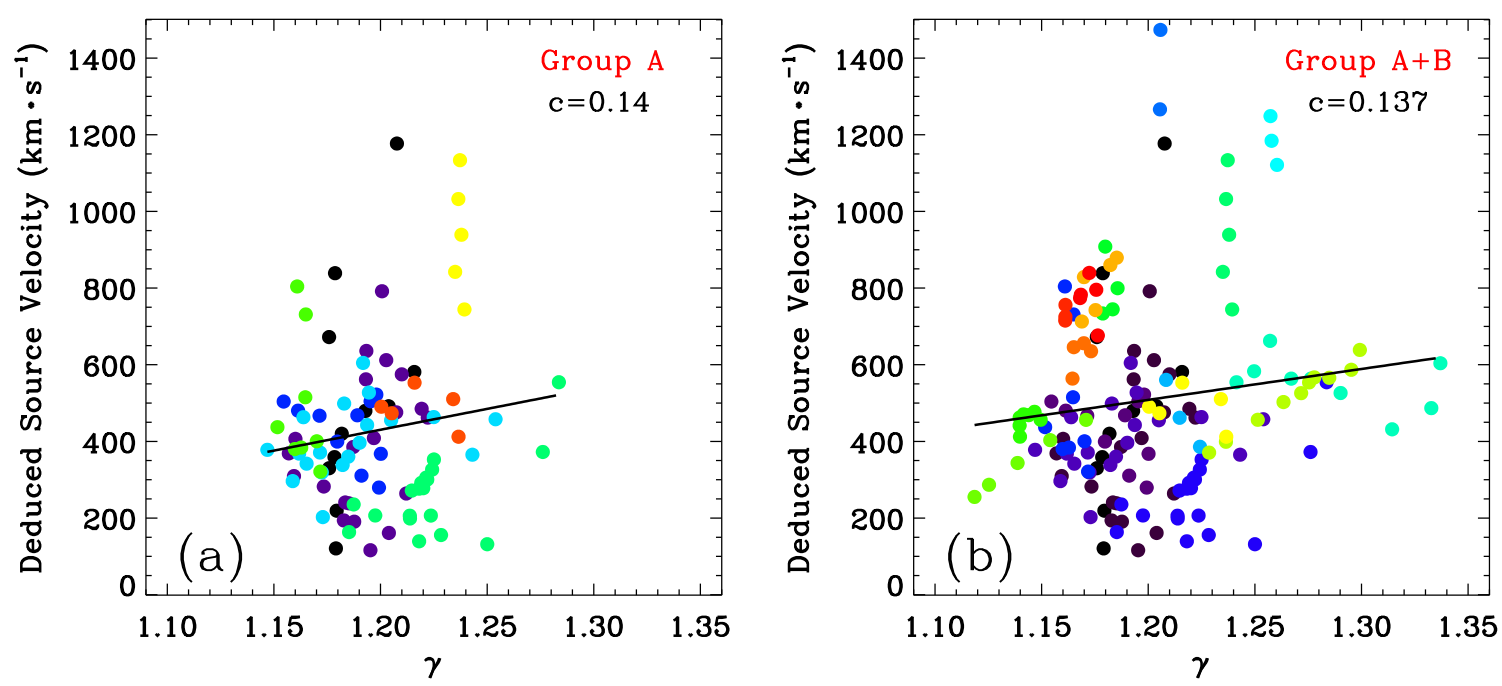

Fig. 4.- Deduced source velocities versus the band-split frequency ratio $(\gamma)$. The velocities are given by fitting the ratio spectra of the lower split band with the $2 \mathrm{X}$ Saito density model. 